\title{
Singlet excited state behavior of uracil and thymine in aqueous solution: a combined experimental and computational study of 11 uracil derivatives
}

\section{Supporting Information}

Thomas Gustavsson*, Akos Banyasz ${ }^{1}$, Elodie Lazzarotto, Dimitra Markovitsi Laboratoire Francis Perrin, CEA/DSM/DRECAM/SPAM - CNRS URA 2453, CEA Saclay, F-91191 Gif-sur-Yvette, France

Giovanni Scalmani, Michael J. Frisch Gaussian, Inc., 340 Quinnipiac St. Bldg 40,Wallingford, CT 06492, USA

\author{
Vincenzo Barone, Roberto Improta* \\ Dipartimento di Chimica, Universita Federico II, Complesso Universitario Monte S. Angelo, Via \\ Cintia, I-80126 Napoli, Italy \\ Istituto Biostrutture e Bioimmagini /CNR, V. Mezzocannone 6-80134 Napoli, Italy
}

AUTHOR EMAIL ADDRESS *: thomas.gustavsson@cea.fr

\footnotetext{
${ }^{1}$ Present Address : Research Institute for Solid State Physics and Optics, Hungarian Academy of Sciences, P.O. Box 49, Budapest, Hungary H 1525.
} 


\section{Notes on the choice of PBEO functional}

We have chosen to use PBE0 hybrid functional in the calculations instead of the more popular B3LYP hybrid functional. Actually,TD-PBE0 is, on the average, judged to be better than TDB3LYP ${ }^{1}$. For example, B3LYP is reported to be good for small molecules but yields to less popular functionals when size increases ${ }^{2}$. The gradient error of PBE0 has been reported to be

lower than the one obtained for B3LYP ${ }^{3}$, which should mean better optimized geometries. A general comparison of hybrid functionals for non bonding interactions was recently presented ${ }^{4}$.

\section{Notes on the lognormal fitting of the steady-state spectroscopic data}

The use of a lognormal function to describe spectroscopic data was originally introduced by Siano and Metzler to characterize absorption spectra ${ }^{5}$. Maroncelli and Fleming applied the lognormal function with success to time-resolved fluorescence spectra ${ }^{6}$, and its use has become a standard method today ${ }^{7}$. We have used the lognormal function for the fitting of the steady-state spectroscopic data. It is given by:

$$
\varepsilon(v)=\varepsilon_{0} \exp \left(-\beta^{2}\left[\ln \frac{v-a}{b}\right]^{2}\right)
$$

An example of a fit is given in figure $\mathrm{S} 1$ below, where the corrected and $\lambda^{2}$-scaled fluorescence spectrum of uracil in aqueous solution is shown on a wavenumber scale. As can be seen, the fit is satisfactory over the whole range and allows a good evaluation of spectroscopic parameters as explained below. 


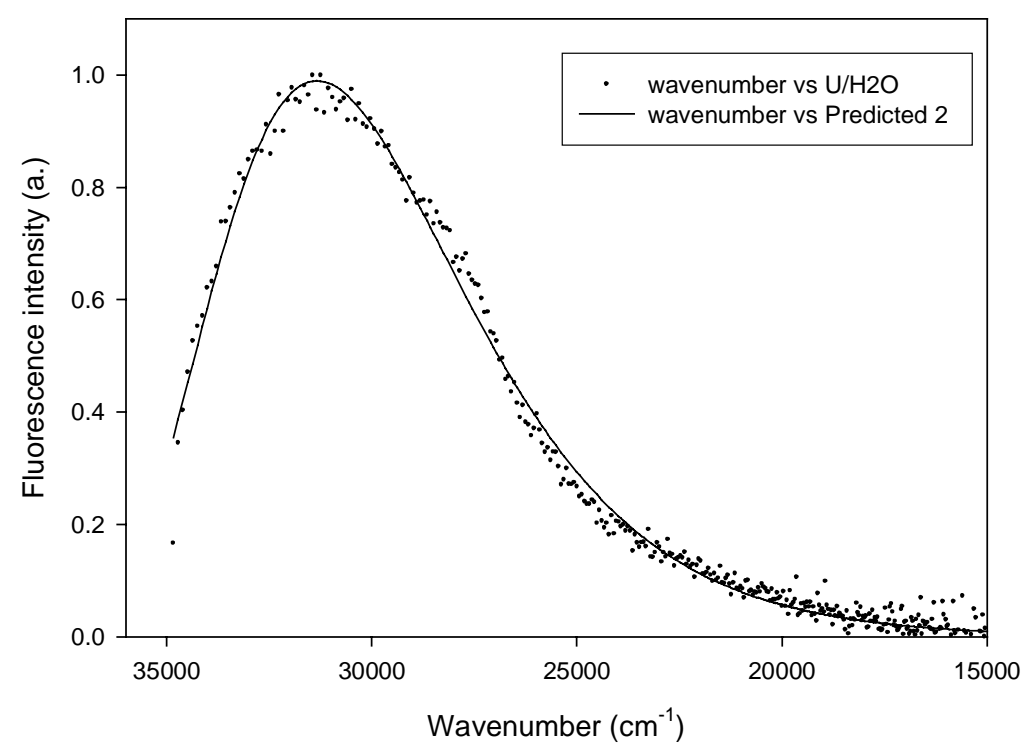

Figure S1. Steady-state fluorescence spectrum of uracil in aqueous solution plotted on wavenumber scale.

Using the parameters obtained from the fit with eq. S1, the peak frequency, mean frequency and the FWHM (in $\mathrm{cm}^{-1}$ ) of the spectrum are given by eqs. S2-S4.

$$
\begin{gathered}
v_{\text {peak }}=a+b \\
v_{\text {mean }}=a+b \exp \left(\frac{3}{4 \beta^{2}}\right) \\
F W H M=2 b \sinh \left(\frac{\sqrt{\ln 2}}{\beta}\right)
\end{gathered}
$$

We have in particular chosen to extrapolate the fluorescence spectrum in the blue wing for the calculations of fluorescence quantum yields. We judge that such an extrapolation is better than using "raw data" that usually are perturbed by the Raman line and/or reabsorption.

Fitted FWHM values $\left(\right.$ in $\mathrm{cm}^{-1}$ ) for the 11 uracil derivatives are given in table $\mathrm{S} 1$ below. 
Table S1. Halfwidths (fwhm) of the absorption and fluorescence bands of the 11 uracil compounds.

\begin{tabular}{l|c|c}
\hline compound & abs $\left(\mathrm{cm}^{-1}\right)$ & fluo $\left(\mathrm{cm}^{-1}\right)$ \\
\hline $\mathrm{U}$ & 4875 & 8132 \\
\hline 1MU & 4582 & 7701 \\
\hline 3MU & 4794 & 7313 \\
\hline 6MU & 4836 & 8842 \\
\hline $\mathrm{T}$ & 5017 & 6912 \\
\hline 1,3DMU & 4488 & 7751 \\
\hline 1MT & 4756 & 5896 \\
\hline 5FU & 5158 & 6152 \\
\hline 5ClU & 4972 & 7933 \\
\hline TFT & 4772 & 7860 \\
\hline 5F1,3DMU & 4807 & 6648 \\
\hline
\end{tabular}

\section{Notes on the fluorescence quantum yield measurements}

The fluorescence quantum yields of uracil and its ten derivatives were determined relative to a reference fluorescence standard. The fluorescence quantum yield is given by the following equation:

$$
\phi_{X}=\frac{A_{X}}{A_{S}} \frac{\left(1-10^{-O D_{S}}\right)}{\left(1-10^{-O D_{X}}\right)} \frac{n_{X}^{2}}{n_{S}^{2}} \phi_{S}
$$

where $O D$ is the absorbance of the solution at the exciting wavelength, $A$ is the area of the fluorescence spectrum and $n$ is the refractive index of the solution. The subscripts $x$ and $s$ denote the studied compound and the fluorescence standard.

Fluorescence spectra were recorded with a SPEX Fluorolog-2 spectrofluorometer. Emission spectra were recorded with a bandpass of $4.71 \mathrm{~nm}$ at excitation side and $2.25 \mathrm{~nm}$ at the emission side. The fluorescence quantum yields were determined by exciting the sample at $255 \mathrm{~nm}$, using 
$1 \mathrm{~cm}$ x $1 \mathrm{~cm}$ quartz cells (QZS). The corresponding optical densities were recorded with a Perkin Lamda 900 spectrophotometer.

The fluorescence quantum yields were determined for different dilute aqueous solutions, the concentrations were varied between $9 \times 10^{-7} \mathrm{M}$ and $8.2 \times 10^{-6} \mathrm{M}$, the corresponding optical densities were within the range: $0.10-0.55$. Quinine sulfate dihydrate / $0.1 \mathrm{M} \mathrm{HClO}_{4}$ were used as reference solution, having the fluorescence quantum yield $\left(\phi_{S}\right)$ of 0.59 .

Since the quantum yield of the fluorescence standard is at least three orders of magnitude higher than the fluorescence quantum yields of the studied compounds, it was impossible to adjust the concentrations so that one could measure the optical densities and the fluorescence intensities of the uracil derivatives and the fluorescence standard under the same conditions. To overcome this problem, at first, a concentrated $\left(O D_{255 \mathrm{~nm}} \approx 1\right)$ quinine sulfate dihydrate / $0.1 \mathrm{M} \mathrm{HClO}_{4}$ solution was prepared and its absorbance was measured then the solution was precisely diluted by a factor of ca. 600 and the fluorescence measurements were performed using the obtained dilute solution. The absorbance of such a dilute solution could not be measured so it was evaluated using the absorbance of the concentrated solution and the factor of dilution. We judged that the error introduced due to the dilution of the quinine sulfate solution is less than $0.5 \%$.

Absorption spectra were recorded between 220 and $400 \mathrm{~nm}$. The absorbances were obtained as the difference of the measured absorbance of the solution and the solvent at the excitation wavelength.

In all cases, we supposed that the studied and the reference solutions were dilute enough that their index of refraction can be considered to be equal $\left(n_{X}^{2} \approx n_{S}^{2}\right)$, therefore their ratio was taken as 1 . 


\section{III.1.Determination of the fluorescence areas:}

In order to determine the area of the fluorescence spectrum it was decomposed as follows:

$$
A=A_{n} \times I_{\text {max }}
$$

where $I_{\max }$ is the maximum intensity of the fluorescence spectrum and $A_{n}$ is the area of the normalized fluorescence spectrum, of which the maximum intensity was normalized to unity. This procedure allowed us to independently measure the maximum intensity $\left(I_{\max }\right)$ and the spectral area to increase experimental precision. The spectral area (shape) was obtained as described in the Experimental section of the article. The measured spectrum was normalized to obtain $A_{n}$.

The fluorescence measurements were performed using dilute solutions therefore the contribution of the solvent emission was not negligible. The fluorescence intensity was measured in the solution and also in the solvent at the wavelength corresponding to the fluorescence maximum of the solution. The maximum intensity of the fluorescence spectrum $\left(I_{\max }\right)$ was evaluated as the difference of the fluorescence intensities measured in the solution and in the solvent:

$$
I_{\max }=I^{\text {solution }}-\alpha \times I^{\text {solvent }}
$$

where $\alpha$ is a scaling factor obtained as the quotient of the Raman intensities $\left(I_{R}\right)$ measured in the solution and the solvent, respectively:

$$
\alpha=I_{R}^{\text {solution }} / I_{R}^{\text {solvent }}
$$

Obviously, $\alpha \leq 1$, because the solute dissolved in the solvent decreases the efficiency of the Raman interaction relative to the pure solvent. It has to be mentioned, that in the case of uracil 
derivatives, the absorption also decreases the Raman intensity, which is close to the absorption maxima of uracil.

\section{III.2.Corrections:}

\section{a. Averaging the absorbance over the full bandwidth:}

The measured optical densities correspond to $1 \mathrm{~nm}$ bandwidth, but the monochromator at the excitation side of the spectrofluorometer had a bandpass of $4.71 \mathrm{~nm}$, so the measured fluorescence signal does not correspond to the measured absorbance. This artifact could be easily avoided by averaging the optical densities over the full excitation bandwidth $(253-257 \mathrm{~nm})$. This correction decreased the optical densities in the case of the studied compounds and the reference solution, decreasing the fluorescence quantum yields by $\leq 2 \%$.

\section{b. Correction for the reabsorption of the Raman emission:}

It was mentioned above that the Raman intensity is used to evaluate the maximum intensity of

the fluorescence spectrum $\left(I_{\max }\right)$. As the measured Raman emission was partly reabsorbed the obtained $I_{\max }$ value is erroneous. This artifact was taken into account in the calculation of the fluorescence quantum yields. Supposing that the Raman emission is generated in the center of the cell and it is attenuated following the Lambert-Beer law, so the corrected Raman intensity can be given as:

$$
I_{R}^{\text {emitted }}=I_{R}^{\text {measured }} \times 10^{O D_{R}} \times 10^{-l / 2}
$$

where $I_{R}^{\text {measured }}$ is the measured Raman intensity, $O D_{R}$ is the measured absorbance at the wavelength of the Raman emission and $l$ is the length of the cell. (One has to note that this artifact can only be observed in aqueous solutions of uracils, because the quinine sulfate solution was so dilute that reabsorption could be neglected.) 
As a result of this correction the maximum intensity of the fluorescence spectrum decreased (see Eq. S5-S8), therefore the quantum yield values also decreased by 1 to $5 \%$ depending on the position of the first absorption band.

\section{c. Calculating the effective intensity deposition:}

Previously, it was supposed that the measured fluorescence intensities correspond to the

intensity depositions $I\left(1-10^{-O D}\right)$ calculated using the optical densities measured in $1 \mathrm{~cm}$ cells. As a matter of fact, the fluorescence intensities correspond to the intensity deposition occurring in a $1.25 \mathrm{~mm}$ region located in the middle of the cell (see Figure $\mathrm{S} 2$ ), because the $1.25 \mathrm{~mm}$ slits let through only this part of the emitted light.

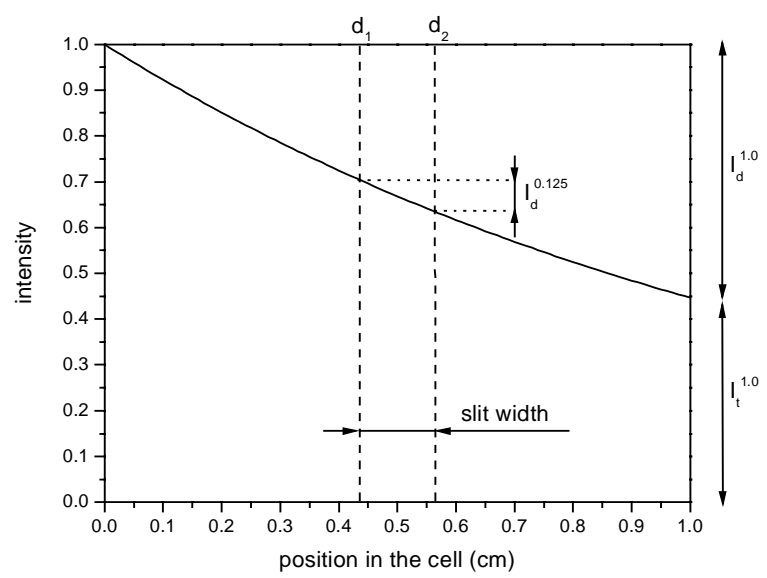

Figure S2. A typical example for the intensity deposition $\left(I_{d}^{1.0}\right)$ occurring in a $1 \mathrm{~cm}$ cell and the intensity deposition $\left(I_{d}^{0.125}\right.$ ) corresponding to the measured fluorescence signal using $1.25 \mathrm{~mm}$ slits, where $d_{1}$ and $d_{2}$ denote the edges of the slit. The absorbance was 0.35 .

The intensity deposition corresponding to the measured fluorescence signal is

$$
I_{d}\left(O D, d_{1}, d_{2}\right)=I\left(10^{-O D d_{1}}-10^{-O D d_{2}}\right)
$$

where $O D$ is the measured absorbance using $1 \mathrm{~cm}$ cell, $d_{1}$ and $d_{2}$ are the edges of the slit. Figure S2 shows that the lower the absorbance, the lower the difference between the intensity 
depositions is. The quinine sulfate reference solution was dilute enough $\left(O D_{255 \mathrm{~nm}} \approx 2 \times 10^{-3}\right)$ that the difference between the intensity depositions was negligible. However, the absorbances of the low fluorescent uracil derivatives had to be kept high enough to get measurable fluorescence signal, so the difference between the intensity depositions cannot be neglected. As a consequence of the above considerations, Eq. S5 can be rewritten as

$$
\phi_{X}^{0.125}=\frac{A_{X}}{A_{S}} \frac{\left(10^{-O D_{S} d_{1}}-10^{-O D_{S} d_{2}}\right)}{\left(10^{-O D_{X} d_{1}}-10^{-O D_{X} d_{2}}\right)} \frac{n_{X}^{2}}{n_{S}^{2}} \phi_{S}
$$

If the Eq. S5 is divided by Eq. S11 the following relation is obtained:

$$
\frac{\phi_{X}^{1.0}}{\phi_{X}^{0.125}}=\frac{\left(10^{-O D_{X} d_{1}}-10^{-O D_{X} d_{2}}\right)}{\left(10^{-O D_{S} d_{1}}-10^{-O D_{S} d_{2}}\right)} \frac{\left(1-10^{-O D_{S}}\right)}{\left(1-10^{-O D_{X}}\right)}
$$

showing the artifact introduced by using the erroneous intensity deposition in the calculation of quantum yields.

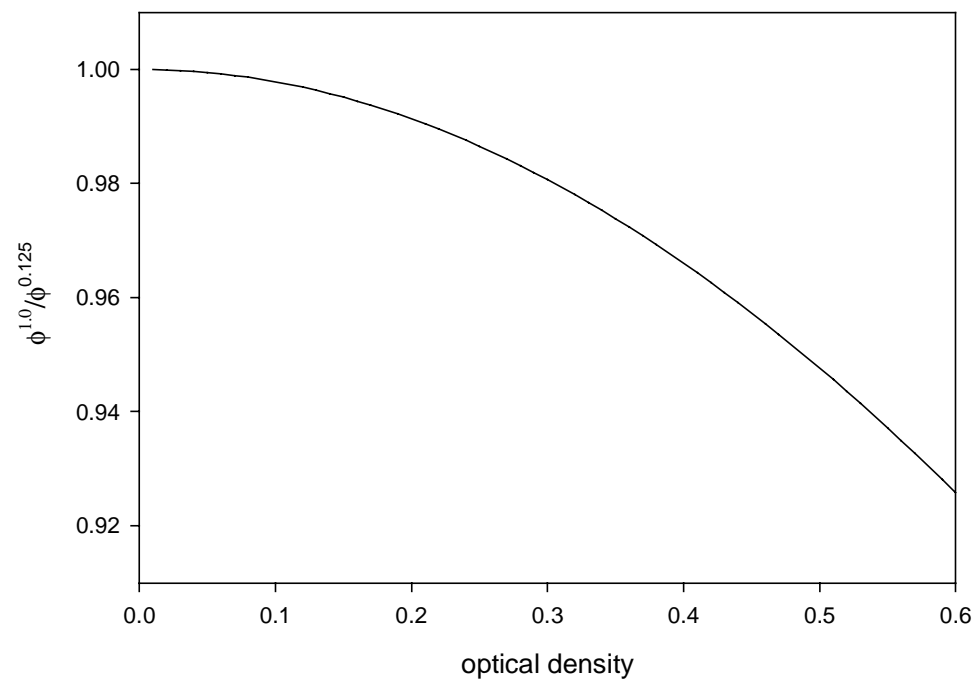

Figure S3. The calculated $\phi_{X}^{1.0} / \phi_{X}^{0.125}$ function versus the absorbance of the studied solution (The absorbance of the reference solution is fixed to $2 \times 10^{-3}$ ). 
The $\phi_{X}^{1.0}$ values we determined decreased with increasing intensity, which can be seen from Figure S3, so we evaluated the fluorescence quantum yields using Eq. S11, to take into account the intensity deposition properly. It has to be noted that in certain cases this correction was not enough to compensate for the decrease of the quantum yields with increasing optical densities. It indicates that there may be some other sources of systematic error which may account for the systematic decrease of fluorescence quantum yields. 


\section{Steady-state and time-resolved spectroscopic data}
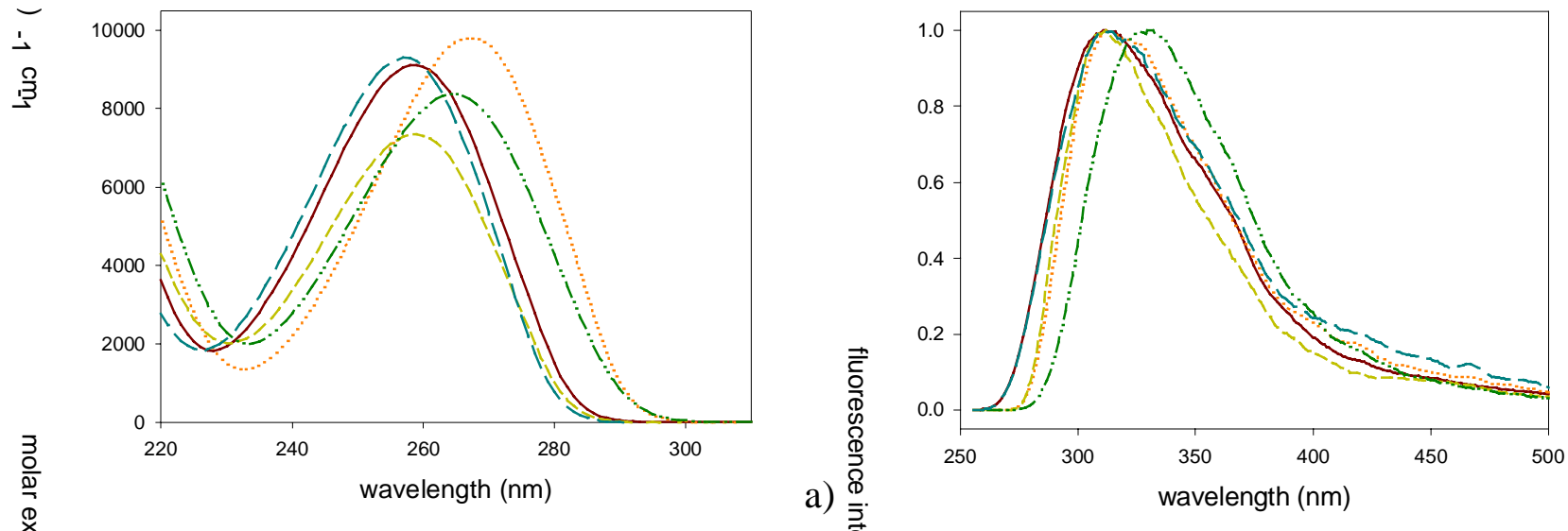

b)

Figure S4. Steady-state absorption a) and fluoresce्ष्तnce b) spectra of monomethylated uracils in $\mathrm{H}_{2} \mathrm{O}$ : uracil, 1-methyluracil, 3-methyluracil, 5-methyluracil (thymine), 6-methyluracil.

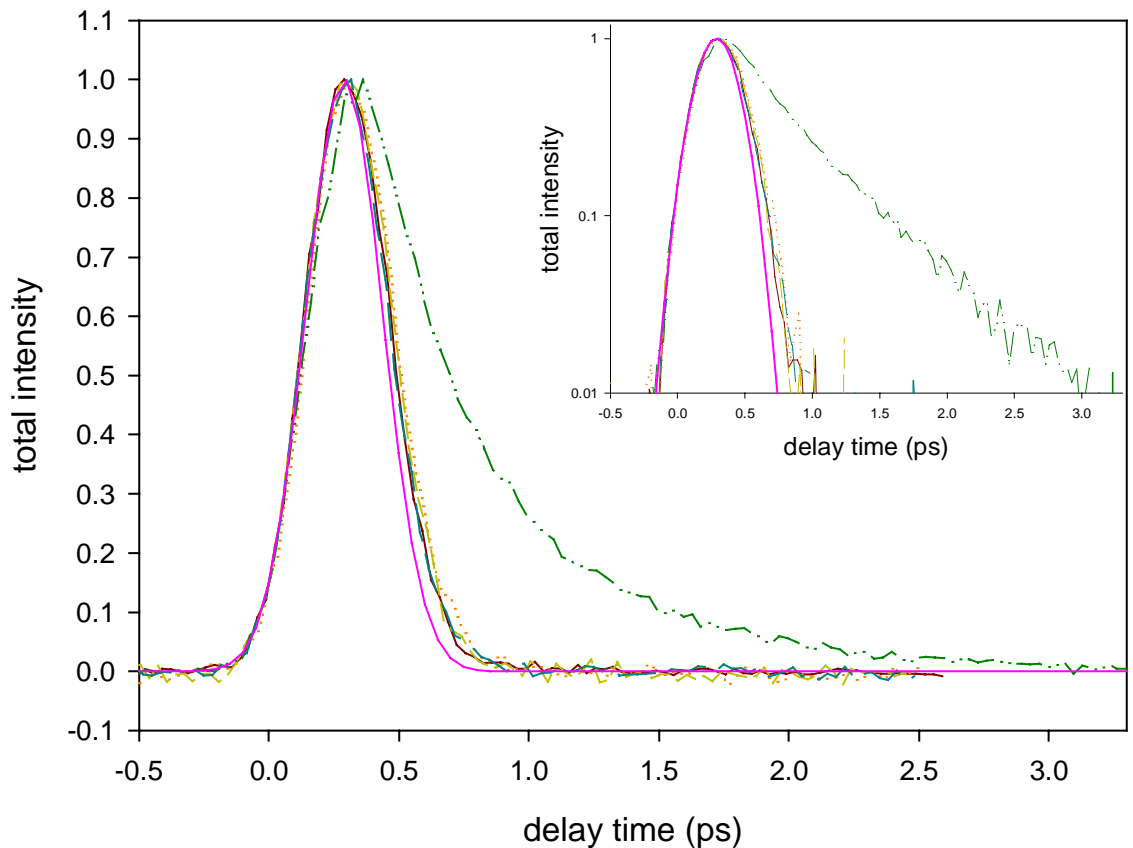

Figure S5. Fluorescence scans recorded for $\sim 2.5 \times 10^{-3} \mathrm{~mol} / \mathrm{dm}^{-3}$ aqueous solutions of uracil and its mono-methylated derivatives: uracil, 1-methyluracil, 3-methyluracil, 5-methyluracil (thymine), 6methyluracil at $330 \mathrm{~nm}$ after excitation at $267 \mathrm{~nm}$. Also shown in this figure is the $330 \mathrm{fs}$ (fwhm)

Gaussian apparatus function. 

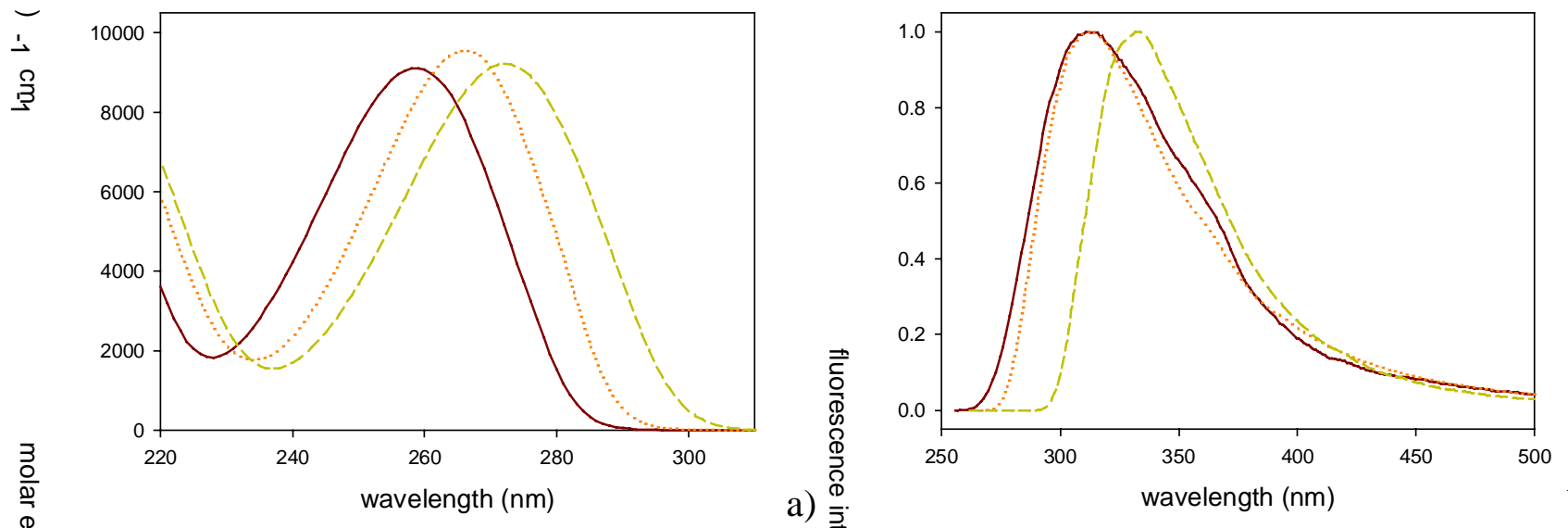

b)

Figure S6. Steady-state absorption a) and fluoresce ${ }^{8}$ ce b) spectra of dimethylated uracils in $\mathrm{H}_{2} \mathrm{O}$ : uracil, 1,3-dimethyluracil, 1-methylthymine.

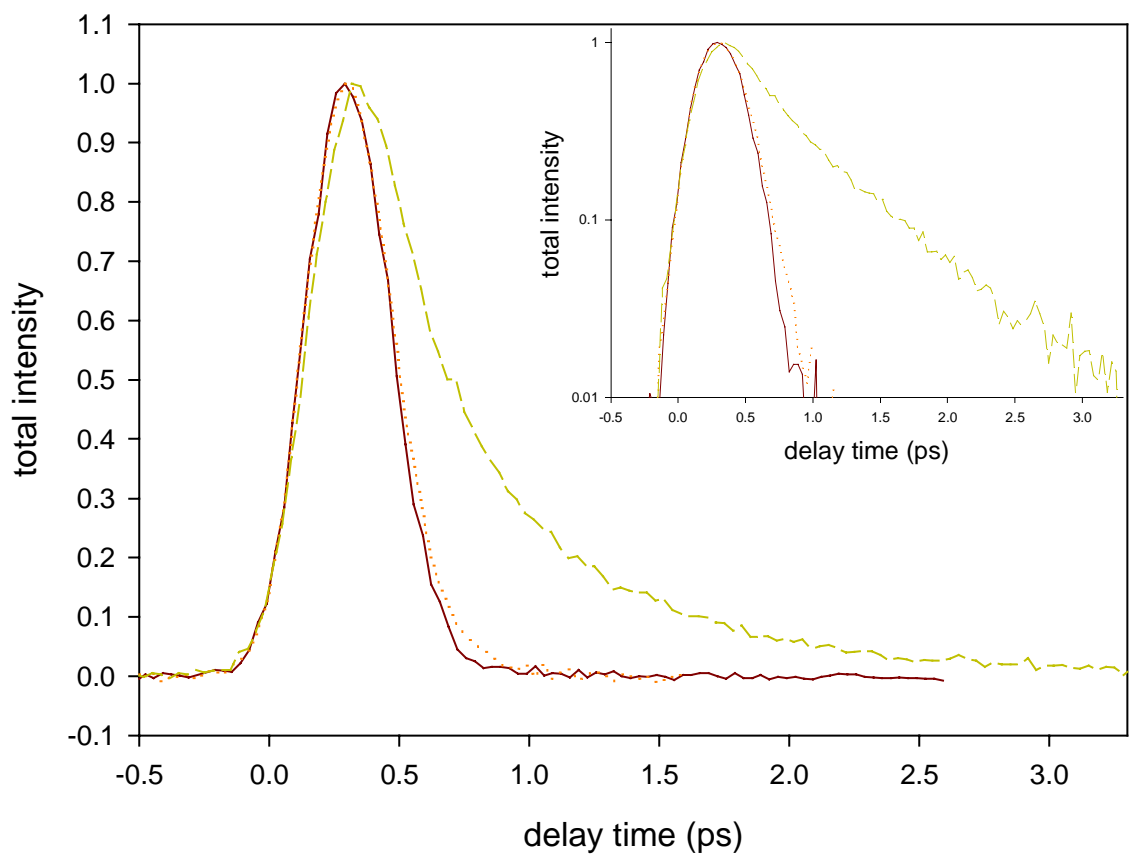

Figure S7. Fluorescence scans recorded for $\sim 2.5 \times 10^{-3} \mathrm{~mol} / \mathrm{dm}^{-3}$ aqueous solutions of uracil and its dimethylated derivatives: uracil, 1,3-dimethyluracil, 1,5-dimethyluracil (1-methylthymine) at $330 \mathrm{~nm}$ after excitation at $267 \mathrm{~nm}$. 

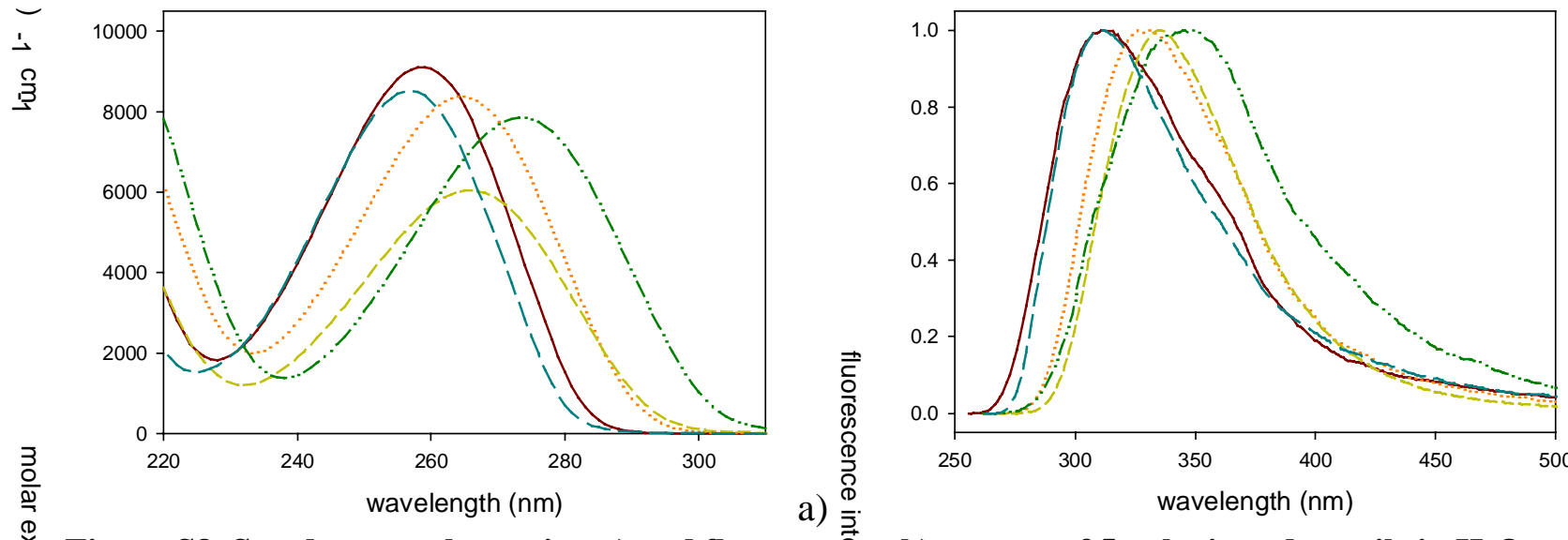

b)

Figure S8. Steady-state absorption a) and fluoresceßnce b) spectra of 5-substituted uracils in $\mathrm{H}_{2} \mathrm{O}$ : uracil, 5-methyluracil (thymine), 5-fluorouraciil, 5-chlorouracil, 5-trifluoromethyluracil (trifluorothymine).

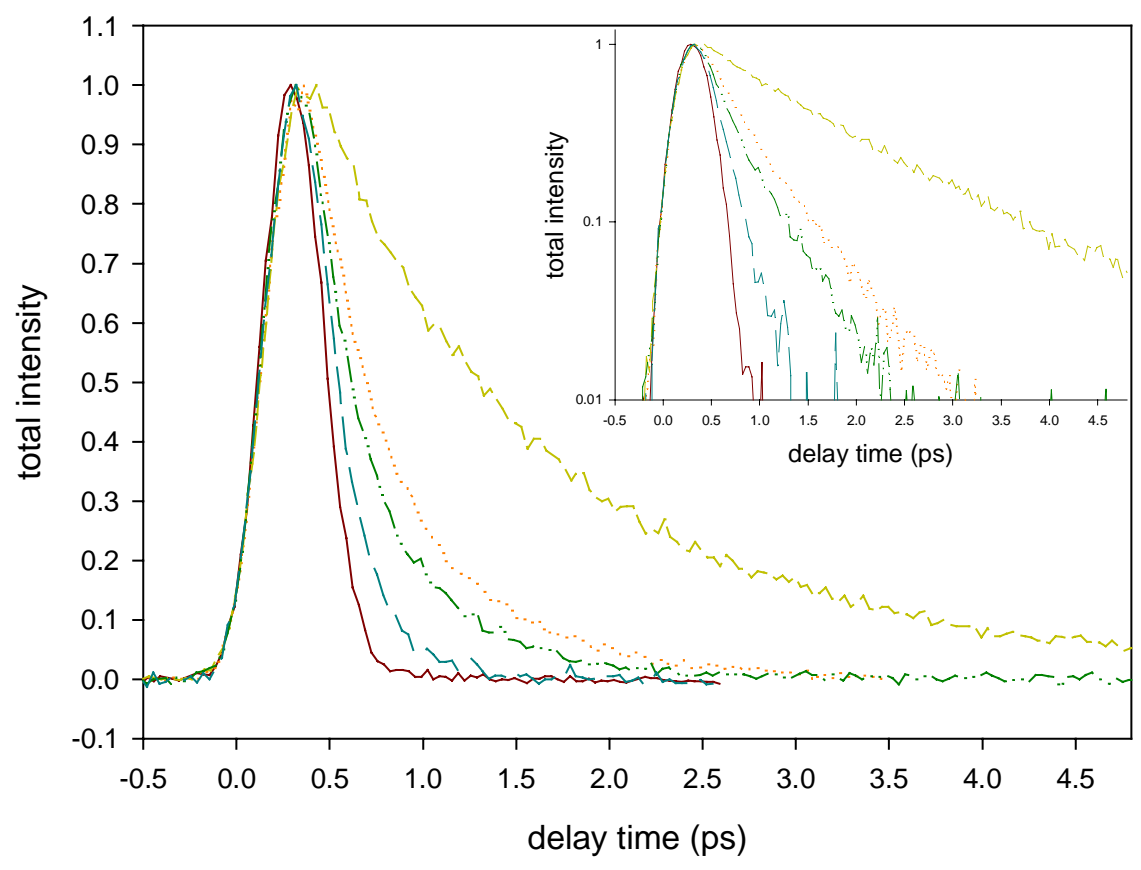

Figure S9. Fluorescence scans recorded for $\sim 2.5 \times 10^{-3} \mathrm{~mol} / \mathrm{dm}^{-3}$ aqueous solutions of uracil and its 5methylated derivatives: uracil, thymine, 5-fluorouracil, 5-chlorouracil, trifluorothymine at $330 \mathrm{~nm}$ after excitation at $267 \mathrm{~nm}$. 

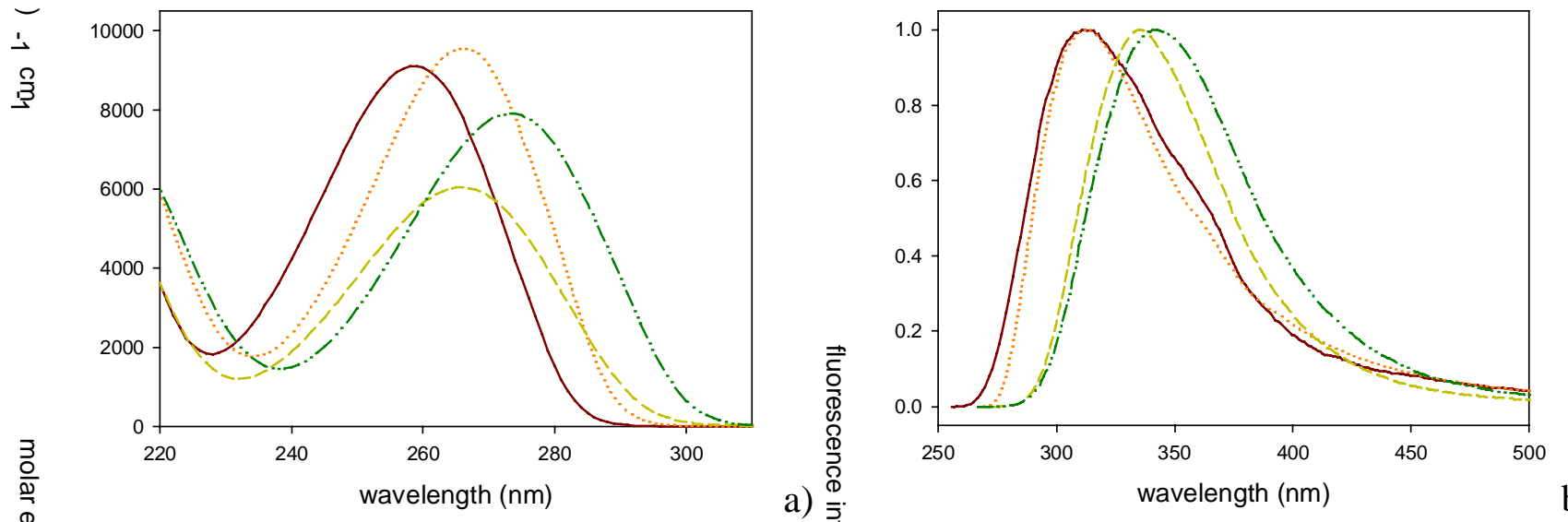

Figure S10. Steady-state absorption a) and fluoresce $\underset{\substack{\frac{N}{*} \\ \gtrless}}{\gtrless}$ b) spectra of 5 -substituted uracils in $\mathrm{H}_{2} \mathrm{O}$ : uracil, 1,3-dimethyluracil, 5-fluorouracil, 5-fluoro-1,3-dimethyluracil.

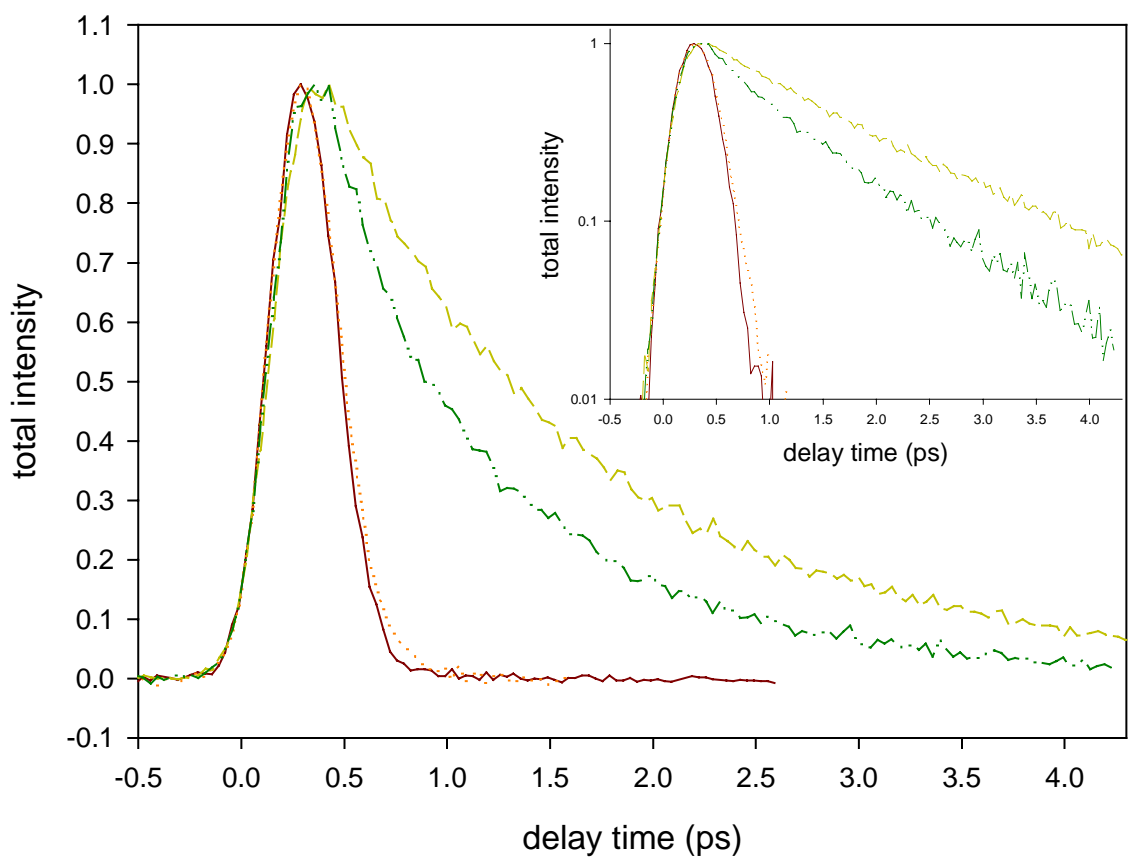

Figure S11. Fluorescence scans recorded for $\sim 2.5 \times 10^{-3} \mathrm{~mol} / \mathrm{dm}^{-3}$ aqueous solutions: uracil, 1,3dimethyluracil, 5-fluorouracil, 5-fluoro-1,3-dimethyluracil at $330 \mathrm{~nm}$ after excitation at $267 \mathrm{~nm}$. 


\section{Additional information regarding the quantum chemical calculations}
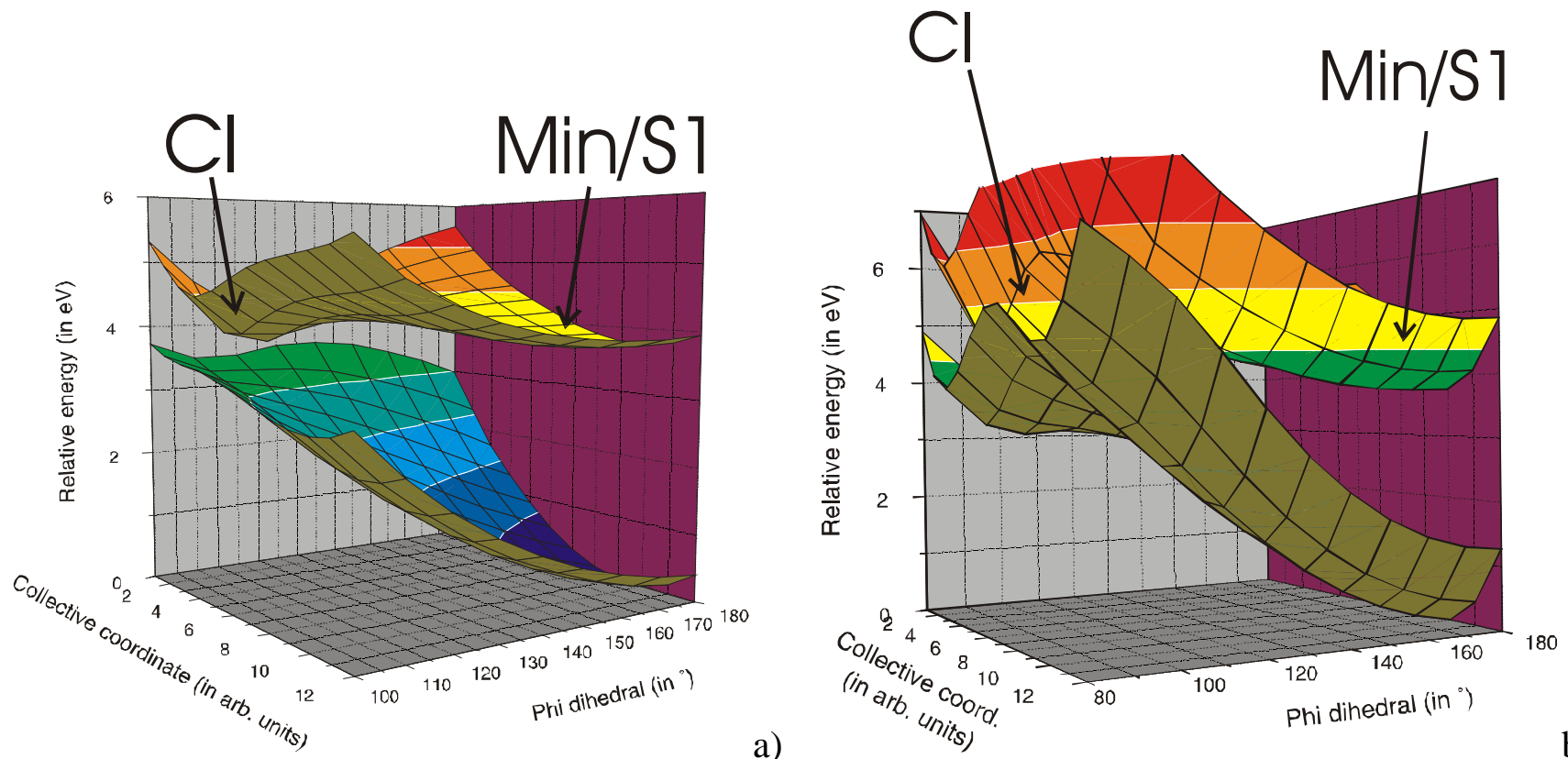

Figure S12. $S_{0}$ and $S_{1}$ potential energy surfaces (in $\mathrm{eV}$ ) in the region connecting the minimum of the $\pi / \pi *$ state and the $S_{0} / S_{1}$ conical intersection for a) thymine and b) 5 -fluorouracil.

Table S2. Cartesian coordinates of the PCM/PBE0/6-31G(d) $S_{0}$ and $S_{1}$ minimum of uracil.

\begin{tabular}{cccc}
\hline \multicolumn{4}{c}{$\mathrm{S}_{0}$} \\
\hline $\begin{array}{c}\text { Atom } \\
\text { Number }\end{array}$ & $\mathrm{x}$ & $\mathrm{y}$ & $\mathrm{z}$ \\
\hline 6 & 0.006963 & 0.010481 & 0.006781 \\
7 & 0.006160 & -0.004889 & 1.370729 \\
6 & 1.155893 & -0.005868 & 2.129086 \\
7 & 2.309309 & 0.008004 & 1.378593 \\
6 & 2.424356 & 0.024345 & -0.012851 \\
6 & 1.155183 & 0.024504 & -0.706896 \\
8 & 1.148210 & -0.016615 & 3.351090 \\
8 & 3.533733 & 0.036468 & -0.537048 \\
1 & 1.149727 & 0.036450 & -1.791892 \\
1 & -0.879740 & -0.013089 & 1.887819 \\
1 & 3.186901 & 0.009362 & 1.912704 \\
1 & -0.978342 & 0.010233 & -0.456582 \\
\hline
\end{tabular}

\begin{tabular}{cccc}
\hline \multicolumn{4}{c}{$\mathrm{S}_{1}$} \\
\hline $\begin{array}{c}\text { Atom } \\
\text { Number }\end{array}$ & $\mathrm{x}$ & $\mathrm{y}$ & $\mathrm{z}$ \\
\hline 7 & -0.077620 & -0.947699 & 0.395812 \\
6 & -1.288017 & -0.277503 & 0.033679 \\
6 & -1.138004 & 1.127066 & -0.085034 \\
6 & 0.138884 & 1.742743 & 0.224494 \\
7 & 1.191267 & 0.935494 & -0.105527 \\
6 & 1.145914 & -0.448354 & 0.012754 \\
8 & -2.311535 & -0.951005 & -0.179446 \\
8 & 2.146704 & -1.128907 & -0.183384 \\
1 & -1.929568 & 1.702908 & -0.566725 \\
1 & 2.110779 & 1.321985 & -0.383510 \\
1 & -0.131904 & -1.972670 & 0.396579 \\
1 & 0.321148 & 2.808793 & 0.308955 \\
\hline
\end{tabular}


Table S3. Cartesian coordinates of the PCM/PBE0/6-31G(d) $S_{0}$ and $S_{1}$ minimum of thymine.

\begin{tabular}{cccc}
\hline \multicolumn{4}{c}{$\mathrm{S}_{0}$} \\
\hline $\begin{array}{c}\text { Atom } \\
\text { Number }\end{array}$ & $\mathrm{x}$ & $\mathrm{y}$ & $\mathrm{z}$ \\
\hline 7 & -1.114026 & -1.235958 & -0.000170 \\
6 & -1.661984 & 0.022722 & -0.000002 \\
7 & -0.719674 & 1.026021 & -0.000053 \\
6 & 0.666366 & 0.892552 & -0.000300 \\
6 & 1.150356 & -0.479408 & -0.000197 \\
6 & 0.232985 & -1.474821 & -0.000272 \\
8 & -2.867349 & 0.232581 & 0.000040 \\
8 & 1.381761 & 1.890228 & 0.000604 \\
6 & 2.626358 & -0.711759 & 0.000024 \\
1 & -1.774351 & -2.020503 & 0.000216 \\
1 & -1.088233 & 1.984689 & 0.000084 \\
1 & 0.510791 & -2.528128 & -0.000333 \\
1 & 3.096077 & -0.256557 & -0.879673 \\
1 & 3.095770 & -0.256981 & 0.880106 \\
1 & 2.855344 & -1.781278 & -0.000205 \\
\hline
\end{tabular}

\begin{tabular}{cccc}
\hline \multicolumn{4}{c}{$\mathrm{S}_{1}$} \\
\hline $\begin{array}{c}\text { Atom } \\
\text { Number }\end{array}$ & $\mathrm{x}$ & $\mathrm{y}$ & $\mathrm{z}$ \\
\hline 6 & -0.673567 & 0.907394 & -0.124511 \\
6 & -1.148087 & -0.439806 & -0.112689 \\
6 & -0.210031 & -1.518170 & -0.341474 \\
7 & 1.072710 & -1.248201 & 0.020989 \\
6 & 1.609439 & 0.030771 & 0.025344 \\
7 & 0.730920 & 1.021917 & -0.356654 \\
8 & 2.786395 & 0.225334 & 0.309466 \\
8 & -1.331007 & 1.937306 & 0.115214 \\
6 & -2.528894 & -0.738511 & 0.316459 \\
1 & 1.746600 & -2.005155 & 0.224188 \\
1 & 1.104709 & 1.971568 & -0.252494 \\
1 & -0.465869 & -2.529572 & -0.645275 \\
1 & -3.100431 & 0.187750 & 0.421821 \\
1 & -3.026778 & -1.410168 & -0.401416 \\
1 & -2.549309 & -1.276343 & 1.280515 \\
\hline & & & \\
\hline
\end{tabular}

Table S4. Cartesian coordinates of the PCM/PBE0/6-31G(d) $S_{0}$ and $S_{1}$ minimum of 5-

\section{fluorouracil.}

\begin{tabular}{cccc}
\hline \multicolumn{4}{c}{$\mathrm{S}_{0}$} \\
\hline $\begin{array}{c}\text { Atom } \\
\text { Number }\end{array}$ & $\mathrm{x}$ & $\mathrm{y}$ & $\mathrm{z}$ \\
\hline 6 & 0.003437 & 0.012113 & 0.004394 \\
7 & 0.003826 & -0.000971 & 1.372089 \\
6 & 1.146044 & -0.009519 & 2.135732 \\
7 & 2.309308 & -0.009808 & 1.389421 \\
6 & 2.443329 & 0.001338 & 0.007083 \\
6 & 1.164113 & 0.012968 & -0.676838 \\
8 & 1.138685 & -0.016671 & 3.356769 \\
8 & 3.537425 & 0.001819 & -0.539705 \\
9 & 1.192843 & 0.024218 & -2.013257 \\
1 & -0.887782 & 0.004063 & 1.880282 \\
1 & 3.183412 & -0.016790 & 1.931245 \\
1 & -0.967765 & 0.020189 & -0.487443 \\
\hline
\end{tabular}

\begin{tabular}{cccc}
\hline \multicolumn{4}{c}{$\mathrm{S}_{1}$} \\
\hline $\begin{array}{c}\text { Atom } \\
\text { Number }\end{array}$ & $\mathrm{x}$ & $\mathrm{y}$ & $\mathrm{z}$ \\
\hline 7 & -0.643956 & 1.015909 & 0.415968 \\
6 & 0.741009 & 0.875723 & 0.094231 \\
6 & 1.136016 & -0.495873 & 0.099431 \\
6 & 0.207911 & -1.566431 & 0.177292 \\
7 & -1.069867 & -1.206566 & -0.143812 \\
6 & -1.567037 & 0.083370 & -0.003150 \\
8 & 1.438715 & 1.854762 & -0.226568 \\
9 & 2.414654 & -0.773290 & -0.134603 \\
8 & -2.749880 & 0.324908 & -0.205210 \\
1 & -1.776356 & -1.923865 & -0.381293 \\
1 & -0.993581 & 1.981696 & 0.423961 \\
1 & 0.416735 & -2.581715 & 0.511064 \\
\hline
\end{tabular}

Table S5. Cartesian coordinates of the PCM/PBE0/6-31G(d) $S_{0}$ minimum of 6-methyluracil.

\begin{tabular}{cccc}
\hline \multicolumn{4}{c}{$\mathrm{S}_{0}$} \\
\hline $\begin{array}{c}\text { Atom } \\
\text { Number }\end{array}$ & $\mathrm{x}$ & $\mathrm{y}$ & $\mathrm{z}$ \\
\hline 6 & 0.003143 & 0.015249 & -0.000072
\end{tabular}




\begin{tabular}{cccc}
7 & 0.000472 & 0.071296 & 1.370837 \\
6 & 1.136899 & 0.065040 & 2.149619 \\
7 & 2.303684 & -0.002839 & 1.428225 \\
6 & 2.435018 & -0.063750 & 0.041007 \\
6 & 1.179861 & -0.051266 & -0.672768 \\
8 & 1.101962 & 0.115717 & 3.370528 \\
8 & 3.551128 & -0.121434 & -0.467723 \\
6 & -1.334108 & 0.032758 & -0.661795 \\
1 & 1.204148 & -0.096309 & -1.757035 \\
1 & -0.880096 & 0.121314 & 1.894471 \\
1 & 3.171221 & -0.009294 & 1.977544 \\
1 & -1.939374 & -0.819078 & -0.329154 \\
1 & -1.880595 & 0.947092 & -0.401067 \\
1 & -1.226512 & -0.015009 & -1.746872 \\
\hline
\end{tabular}

Table S6. Cartesian coordinates of the PCM/PBE0/6-31G(d) $S_{0}$ and $S_{1}$ minimum of 1,3dimethyluracil.

\begin{tabular}{cccc}
\hline \multicolumn{4}{c}{$\mathrm{S}_{0}$} \\
\hline $\begin{array}{c}\text { Atom } \\
\text { Number }\end{array}$ & $\mathrm{x}$ & $\mathrm{y}$ & $\mathrm{z}$ \\
\hline 6 & -0.006962 & 0.000302 & 0.001486 \\
7 & 0.004674 & 0.012989 & 1.407857 \\
6 & 1.138509 & 0.015748 & 2.197870 \\
7 & 2.349620 & 0.005104 & 1.527845 \\
6 & 2.409386 & -0.007655 & 0.164802 \\
6 & 1.300392 & -0.010481 & -0.605563 \\
6 & -1.262067 & 0.024545 & 2.123668 \\
8 & 1.083890 & 0.026935 & 3.419724 \\
6 & 3.549948 & 0.008365 & 2.351398 \\
8 & -1.067883 & -0.000962 & -0.616605 \\
1 & 3.567864 & -0.874307 & 2.995534 \\
1 & 3.573862 & 0.902250 & 2.979666 \\
1 & 4.419792 & -0.000440 & 1.693171 \\
1 & 3.411769 & -0.015399 & -0.261478 \\
1 & 1.367223 & -0.020638 & -1.688607 \\
1 & -2.054589 & 0.020298 & 1.377810 \\
1 & -1.338705 & -0.857225 & 2.764757 \\
1 & -1.332220 & 0.919053 & 2.747640 \\
\hline
\end{tabular}

\begin{tabular}{cccc}
\hline \multicolumn{4}{c}{$\mathrm{S}_{1}$} \\
\hline $\begin{array}{c}\text { Atom } \\
\text { Number }\end{array}$ & $\mathrm{x}$ & $\mathrm{y}$ & $\mathrm{z}$ \\
\hline 6 & -0.012807 & 0.053369 & -0.004949 \\
7 & 0.008828 & 0.113558 & 1.367280 \\
6 & 1.258945 & 0.043070 & 2.069473 \\
6 & 2.254306 & -0.707603 & 1.396214 \\
6 & 1.939863 & -1.376260 & 0.166120 \\
7 & 1.050213 & -0.642629 & -0.602250 \\
8 & 1.376582 & 0.624080 & 3.161120 \\
8 & -0.899809 & 0.547920 & -0.691317 \\
1 & 3.273565 & -0.690424 & 1.784236 \\
6 & 1.169502 & -0.632706 & -2.043356 \\
6 & -1.109935 & 0.777014 & 2.013117 \\
1 & 2.627184 & -2.024231 & -0.369118 \\
1 & 0.396171 & 0.006863 & -2.465090 \\
1 & 2.167241 & -0.272038 & -2.319400 \\
1 & 1.057053 & -1.658561 & -2.419331 \\
1 & -2.043982 & 0.360617 & 1.632559 \\
1 & -1.025958 & 0.598650 & 3.084407 \\
1 & -1.102103 & 1.857796 & 1.831634 \\
\hline
\end{tabular}

Table S7. Cartesian coordinates of the PCM/PBE0/6-31G(d) S0 and $S_{1}$ minimum of the adduct between uracil and four water molecules.

\begin{tabular}{cccc}
\hline \multicolumn{5}{c}{$\mathrm{S}_{0}$} \\
\hline $\begin{array}{c}\text { Atom } \\
\text { Number }\end{array}$ & $\mathrm{x}$ & $\mathrm{y}$ & $\mathrm{z}$ \\
\hline 7 & -0.034527 & -0.045749 & 0.051384
\end{tabular}

\begin{tabular}{cccc}
\hline \multicolumn{4}{c}{$\mathrm{S}_{1}$} \\
\hline $\begin{array}{c}\text { Atom } \\
\text { Number }\end{array}$ & $\mathrm{x}$ & $\mathrm{y}$ & $\mathrm{z}$ \\
\hline 7 & 2.001300 & -0.404197 & -0.118824
\end{tabular}




\begin{tabular}{cccccccc}
6 & -0.012836 & -0.088461 & 1.419283 & 6 & 0.897145 & 0.395916 & 0.076566 \\
7 & 1.227435 & -0.072560 & 1.990712 & 7 & -0.329389 & -0.198188 & -0.082925 \\
6 & 2.433607 & 0.034621 & 1.311024 & 6 & -0.477060 & -1.602172 & 0.062110 \\
6 & 2.326802 & 0.061481 & -0.126604 & 6 & 0.696609 & -2.355047 & -0.164441 \\
6 & 1.100367 & 0.020967 & -0.697577 & 6 & 1.920563 & -1.696293 & -0.564533 \\
8 & -1.059235 & -0.139587 & 2.080490 & 8 & 1.046432 & 1.590365 & 0.358299 \\
8 & 3.499206 & 0.098069 & 1.935841 & 8 & -1.596249 & -2.077487 & 0.385688 \\
8 & 1.300089 & -0.293461 & 4.719977 & 1 & 2.936569 & 0.054779 & 0.027435 \\
8 & 3.615543 & 1.112450 & 4.534378 & 1 & -1.142996 & 0.390494 & 0.172536 \\
8 & -1.116573 & 0.962265 & 4.670276 & 1 & 0.689278 & -3.421014 & 0.060547 \\
8 & -2.778486 & -0.116210 & -0.234271 & 1 & 2.853972 & -2.209770 & -0.771354 \\
1 & 3.232388 & 0.125938 & -0.721138 & 8 & 4.506883 & 0.617203 & 0.191611 \\
1 & -0.981366 & -0.054082 & -0.365095 & 1 & 4.574406 & 1.459827 & -0.300225 \\
1 & 1.262225 & -0.174251 & 3.039230 & 1 & 4.632143 & 0.882733 & 1.124530 \\
1 & 0.946302 & 0.046546 & -1.775393 & 1 & -0.262170 & 2.939626 & -0.316887 \\
1 & 3.657649 & 0.761144 & 3.617765 & 8 & -1.047580 & 3.414980 & -0.632541 \\
1 & 4.290619 & 0.597099 & 5.013959 & 1 & -1.764381 & 2.825141 & -0.335295 \\
1 & 2.078221 & 0.297382 & 4.857721 & 8 & -2.610571 & 1.374574 & 0.485897 \\
1 & 0.495294 & 0.247851 & 4.897665 & 1 & -3.257434 & 0.666415 & 0.214557 \\
1 & -2.663856 & -0.135425 & 0.734644 & 1 & -2.731074 & 1.476634 & 1.449050 \\
1 & -3.125062 & 0.779944 & -0.409108 & 1 & -3.138414 & -1.337154 & -0.016554 \\
1 & -1.204387 & 0.591733 & 3.767106 & 8 & -3.956142 & -0.810774 & -0.188418 \\
1 & -1.718400 & 0.413769 & 5.207445 & 1 & -4.058992 & -0.836328 & -1.158591 \\
\hline
\end{tabular}

Table S8. Cartesian coordinates of the PCM/PBE0/6-31G(d) $S_{0}$ and $S_{1}$ minimum of the adduct between thymine and four water molecules.

\begin{tabular}{cccc}
\hline \multicolumn{4}{c}{$\mathrm{S}_{0}$} \\
\hline $\begin{array}{c}\text { Atom } \\
\text { Number }\end{array}$ & $\mathrm{x}$ & $\mathrm{y}$ & $\mathrm{z}$ \\
\hline 7 & -0.026427 & -0.054567 & 0.041913 \\
6 & -0.016865 & -0.074909 & 1.406311 \\
7 & 1.219974 & -0.062659 & 1.982460 \\
6 & 2.427180 & 0.023166 & 1.306730 \\
6 & 2.348389 & 0.028599 & -0.141036 \\
6 & 1.116142 & -0.010875 & -0.703801 \\
8 & -1.068157 & -0.103405 & 2.065753 \\
8 & 3.491501 & 0.087296 & 1.933445 \\
8 & 1.287671 & -0.300962 & 4.713297 \\
8 & 3.621760 & 1.084100 & 4.535853 \\
8 & -1.112507 & 0.994459 & 4.652873 \\
8 & -2.781350 & -0.140615 & -0.195550 \\
6 & 3.618317 & 0.092000 & -0.926854 \\
1 & -0.967664 & -0.061570 & -0.382977 \\
1 & 1.252837 & -0.150914 & 3.031549 \\
1 & 0.962165 & -0.001722 & -1.782352 \\
1 & 3.657054 & 0.727723 & 3.620748 \\
1 & 4.293879 & 0.565356 & 5.015762 \\
1 & 2.075969 & 0.274304 & 4.856403 \\
1 & 0.492692 & 0.254437 & 4.889109
\end{tabular}

\begin{tabular}{cccc}
\hline \multicolumn{4}{c}{$\mathrm{S}_{1}$} \\
\hline $\begin{array}{c}\text { Atom } \\
\text { Number }\end{array}$ & $\mathrm{x}$ & $\mathrm{y}$ & $\mathrm{z}$ \\
\hline 7 & -0.016996 & 0.009054 & 0.017102 \\
6 & -0.002461 & -0.013563 & 1.393258 \\
7 & 1.226679 & 0.091166 & 1.997398 \\
6 & 2.339318 & 0.661748 & 1.326482 \\
6 & 2.268897 & 0.652340 & -0.094962 \\
6 & 1.107552 & 0.083501 & -0.745048 \\
8 & -1.058053 & -0.150534 & 2.021704 \\
8 & 3.276523 & 1.158030 & 2.005302 \\
1 & -0.955633 & -0.109747 & -0.435486 \\
1 & 1.203903 & 0.193994 & 3.027274 \\
6 & 3.305802 & 1.316327 & -0.906550 \\
1 & 1.007967 & -0.056010 & -1.816976 \\
8 & -2.363164 & -0.314260 & -1.350403 \\
1 & -2.935429 & 0.462686 & -1.192438 \\
1 & -2.865922 & -1.055269 & -0.957556 \\
1 & -1.092145 & -1.104540 & 3.758144 \\
8 & -0.909432 & -1.492509 & 4.629608 \\
1 & -0.116550 & -0.993897 & 4.899571 \\
8 & 1.268756 & 0.257133 & 4.829045 \\
1 & 1.030840 & 1.154364 & 5.130186
\end{tabular}




\begin{tabular}{|c|c|c|c|c|c|c|c|}
\hline 1 & -2.596609 & -0.111652 & 0.763477 & 1 & 2.263147 & 0.217483 & 4.883109 \\
\hline 1 & -3.166969 & 0.735594 & -0.386245 & 1 & 3.757485 & 0.575770 & 3.578520 \\
\hline 1 & -1.197092 & 0.611560 & 3.754039 & 8 & 3.903122 & 0.266866 & 4.506405 \\
\hline 1 & -1.719077 & 0.456037 & 5.194787 & 1 & 4.210148 & -0.654214 & 4.407819 \\
\hline 1 & 3.413187 & 0.095320 & -2.001080 & 1 & 2.895544 & 2.186828 & -1.447835 \\
\hline 1 & 4.186988 & 0.995244 & -0.678296 & 1 & 3.678666 & 0.638492 & -1.691608 \\
\hline 1 & 4.264098 & -0.763649 & -0.698983 & 1 & 4.133240 & 1.653034 & -0.278295 \\
\hline
\end{tabular}

Table S9. Cartesian coordinates of the PCM/PBE0/6-31G(d) $S_{0}$ and $S_{1}$ minimum of the adduct between 5-fluorouracil and four water molecules.

\begin{tabular}{cccc}
\hline \multicolumn{4}{c}{$\mathrm{S}_{0}$} \\
\hline $\begin{array}{c}\text { Atom } \\
\text { Number }\end{array}$ & $\mathrm{x}$ & $\mathrm{y}$ & $\mathrm{z}$ \\
\hline 7 & 1.969690 & -0.380627 & -0.010984 \\
6 & 0.953378 & 0.511734 & -0.198787 \\
7 & -0.304370 & -0.031323 & -0.279293 \\
6 & -0.628449 & -1.365497 & -0.134649 \\
6 & 0.517195 & -2.227864 & 0.052199 \\
6 & 1.766015 & -1.723796 & 0.109431 \\
8 & 1.165479 & 1.726643 & -0.291703 \\
8 & -1.794047 & -1.767940 & -0.166355 \\
8 & -2.332196 & 1.727128 & -0.728146 \\
8 & -3.906767 & -0.071279 & 0.564650 \\
8 & -0.799676 & 3.513463 & 0.628863 \\
8 & 4.100589 & 1.337393 & 0.044750 \\
9 & 0.282121 & -3.536892 & 0.177052 \\
1 & 2.914117 & 0.045931 & 0.054552 \\
1 & -1.093882 & 0.639791 & -0.493909 \\
1 & 2.646514 & -2.347146 & 0.258486 \\
1 & -3.227357 & -0.738706 & 0.329278 \\
1 & -4.644615 & -0.264698 & -0.043456 \\
1 & -3.011160 & 1.258488 & -0.186784 \\
1 & -1.966698 & 2.453009 & -0.167706 \\
1 & 3.420336 & 2.027464 & -0.064778 \\
1 & 4.426597 & 1.475584 & 0.955530 \\
1 & -0.034091 & 2.969011 & 0.348490 \\
1 & -0.741971 & 4.306228 & 0.063131 \\
\hline & & &
\end{tabular}

\begin{tabular}{cccc}
\hline \multicolumn{5}{c}{$\mathrm{S}_{1}$} \\
\hline $\begin{array}{c}\text { Atom } \\
\text { Number }\end{array}$ & $\mathrm{x}$ & $\mathrm{y}$ & $\mathrm{z}$ \\
\hline 6 & 0.776279 & -2.000604 & -0.212293 \\
6 & 1.998456 & -1.309565 & -0.399095 \\
7 & 1.970429 & -0.000640 & -0.012558 \\
6 & 0.807827 & 0.731058 & 0.119006 \\
7 & -0.374519 & 0.062134 & -0.076537 \\
6 & -0.457284 & -1.347253 & 0.072082 \\
8 & 0.868791 & 1.937711 & 0.367687 \\
8 & -1.500505 & -1.912504 & 0.490820 \\
9 & 0.796569 & -3.325243 & -0.191066 \\
8 & 4.377208 & 1.251925 & 0.183099 \\
8 & -3.945797 & -0.858797 & -0.115408 \\
8 & -2.765208 & 1.454831 & 0.409587 \\
8 & -1.346846 & 3.605186 & -0.704188 \\
1 & 2.868012 & 0.539389 & 0.082453 \\
1 & -1.233767 & 0.605043 & 0.134996 \\
1 & 2.890432 & -1.713740 & -0.873489 \\
1 & 4.362505 & 2.055935 & -0.373751 \\
1 & 4.477926 & 1.600556 & 1.091413 \\
1 & -0.534178 & 3.200179 & -0.362587 \\
1 & -2.021643 & 2.960987 & -0.422836 \\
1 & -2.882442 & 1.578585 & 1.370847 \\
1 & -3.354502 & 0.684864 & 0.173594 \\
1 & -3.091946 & -1.321452 & 0.069067 \\
1 & -4.073714 & -0.962249 & -1.077438 \\
\hline & & &
\end{tabular}

Table S10. Cartesian coordinates of the PCM/PBE0/6-31G(d) $S_{0}$ minimum of the adduct between 6-methyluracil and four water molecules.

\begin{tabular}{cccc}
\hline \multicolumn{4}{c}{$\mathrm{S}_{0}$} \\
\hline $\begin{array}{c}\text { Atom } \\
\text { Number }\end{array}$ & $\mathrm{x}$ & $\mathrm{y}$ & $\mathrm{z}$ \\
\hline 7 & 1.734560 & 0.140710 & -0.052450 \\
6 & 0.492430 & 0.699980 & -0.191720
\end{tabular}




\begin{tabular}{cccc}
7 & -0.548650 & -0.185950 & -0.250880 \\
6 & -0.443330 & -1.562930 & -0.133390 \\
6 & 0.896780 & -2.068560 & -0.003630 \\
6 & 1.948040 & -1.207590 & 0.035680 \\
8 & 0.340710 & 1.923340 & -0.259470 \\
8 & -1.460630 & -2.270840 & -0.143240 \\
8 & -3.038140 & 0.879990 & -0.720470 \\
8 & -3.945210 & -1.289800 & 0.647860 \\
8 & -2.069070 & 3.068250 & 0.580610 \\
8 & 3.982800 & 1.753020 & 0.097320 \\
1 & 1.040950 & -3.141690 & 0.074140 \\
1 & 2.537600 & 0.799160 & 0.004860 \\
1 & -1.500530 & 0.220070 & -0.436430 \\
6 & 3.370560 & -1.633650 & 0.190270 \\
1 & -3.087420 & -1.698150 & 0.393650 \\
1 & -4.589740 & -1.712020 & 0.050100 \\
1 & -3.538090 & 0.240710 & -0.160580 \\
1 & -2.905210 & 1.693020 & -0.178550 \\
1 & 3.886150 & 2.464180 & -0.566040 \\
1 & 3.979970 & 2.230530 & 0.949700 \\
1 & -1.183320 & 2.735480 & 0.318210 \\
1 & -2.228240 & 3.811540 & -0.030480 \\
1 & 3.988170 & -1.214870 & -0.611520 \\
1 & 3.777870 & -1.264340 & 1.138760 \\
1 & 3.449850 & -2.722160 & 0.173470 \\
\hline & & &
\end{tabular}

Table S11. Cartesian coordinates of the PCM/PBE0/6-31G(d) $S_{0}$ and $S_{1}$ minimum of the adduct between 1,3-dimethyluracil and four water molecules.

\begin{tabular}{cccc}
\hline \multicolumn{4}{c}{$\mathrm{S}_{0}$} \\
\hline $\begin{array}{c}\text { Atom } \\
\text { Number }\end{array}$ & $\mathrm{x}$ & $\mathrm{y}$ & $\mathrm{z}$ \\
\hline 6 & -1.419623 & -0.357304 & -0.063755 \\
7 & -0.224253 & 0.363752 & -0.124466 \\
6 & 1.029060 & -0.199209 & -0.010282 \\
7 & 1.089717 & -1.560221 & 0.180729 \\
6 & -0.045340 & -2.312564 & 0.260079 \\
6 & -1.276267 & -1.768851 & 0.147786 \\
6 & -0.251304 & 1.808151 & -0.350330 \\
8 & 2.051846 & 0.484064 & -0.080319 \\
6 & 2.410311 & -2.160935 & 0.355390 \\
8 & -2.511906 & 0.212579 & -0.189637 \\
8 & -3.532885 & 2.877378 & -0.441247 \\
8 & 2.370543 & 2.852152 & 1.562567 \\
1 & 2.811804 & -1.904251 & 1.340039 \\
1 & 3.093424 & -1.795243 & -0.413009 \\
1 & 2.303028 & -3.243082 & 0.272631 \\
1 & 0.104537 & -3.380059 & 0.416887 \\
1 & -2.175087 & -2.371389 & 0.206965 \\
1 & 2.375018 & 2.067767 & 0.988386
\end{tabular}

\begin{tabular}{cccc}
\hline \multicolumn{4}{c}{$\mathrm{S}_{1}$} \\
\hline $\begin{array}{c}\text { Atom } \\
\text { Number }\end{array}$ & $\mathrm{x}$ & $\mathrm{y}$ & $\mathrm{z}$ \\
\hline 7 & -0.245227 & 0.375582 & -0.329336 \\
6 & -1.491431 & -0.305002 & -0.198547 \\
6 & -1.423923 & -1.707263 & -0.350099 \\
6 & -0.178092 & -2.325516 & -0.683760 \\
7 & 0.904248 & -1.673486 & -0.126882 \\
6 & 0.920240 & -0.280368 & -0.016024 \\
8 & -2.524478 & 0.357405 & 0.063647 \\
6 & 2.041755 & -2.450498 & 0.326417 \\
8 & 1.948864 & 0.302542 & 0.331361 \\
6 & -0.248689 & 1.828817 & -0.265024 \\
8 & -4.587713 & -1.542866 & 0.405580 \\
8 & -3.320245 & 3.102421 & 0.194024 \\
8 & 4.797191 & -0.309709 & 0.142067 \\
8 & 3.032014 & 2.980876 & -0.158501 \\
1 & -2.308858 & -2.295035 & -0.106179 \\
1 & -0.049674 & -3.370004 & -0.950008 \\
1 & 2.875927 & -1.803964 & 0.590369 \\
1 & 1.739474 & -3.052383 & 1.192454
\end{tabular}




\begin{tabular}{cccccccc}
1 & 2.594623 & 3.576390 & 0.951416 & 1 & 2.337020 & -3.131219 & -0.481019 \\
1 & -3.267615 & 1.945113 & -0.355207 & 1 & 0.695942 & 2.208489 & -0.648079 \\
1 & -3.527683 & 3.018715 & -1.404658 & 1 & -1.074116 & 2.202681 & -0.868133 \\
1 & -1.285812 & 2.143000 & -0.330432 & 1 & -0.388207 & 2.182539 & 0.762759 \\
1 & 0.322957 & 2.311853 & 0.430610 & 1 & 2.683280 & 2.093162 & 0.013320 \\
1 & 0.195426 & 2.034077 & -1.322635 & 1 & 3.861558 & -0.056309 & 0.161795 \\
1 & 3.844354 & 0.158670 & -0.734444 & 1 & 2.807049 & 3.468071 & 0.654537 \\
8 & 4.675775 & -0.116765 & -1.155804 & 1 & 5.152043 & 0.098861 & 0.952136 \\
1 & 4.486447 & 0.009714 & -2.103582 & 1 & -3.942343 & -0.819475 & 0.276465 \\
1 & -3.947127 & -1.024904 & -0.089368 & 1 & -3.012802 & 2.179391 & 0.156261 \\
8 & -4.555293 & -1.753994 & 0.132277 & 1 & -4.576082 & -1.676882 & 1.369997 \\
1 & -4.652190 & -1.670133 & 1.096538 & 1 & -3.897575 & 3.171036 & -0.586350 \\
\hline
\end{tabular}

Table S12. Cartesian coordinates of the $\operatorname{CASSCF}(8 / 8) / 6-31 G(d)$ optimized structure of uracil and 5-fluorouracil.

\begin{tabular}{cccc}
\hline \multicolumn{4}{c}{ uracil } \\
\hline $\begin{array}{c}\text { Atom } \\
\text { Number }\end{array}$ & $\mathrm{x}$ & $\mathrm{y}$ & $\mathrm{z}$ \\
\hline 7 & -1.239185 & 0.934059 & -0.060253 \\
6 & -1.130022 & -0.486715 & 0.034141 \\
7 & 0.132333 & -0.969691 & -0.151377 \\
6 & 1.346727 & -0.223728 & -0.011821 \\
6 & 1.089241 & 1.188845 & 0.369076 \\
6 & -0.147606 & 1.663413 & -0.215736 \\
8 & -2.122177 & -1.141806 & 0.144994 \\
8 & 2.378100 & -0.821881 & -0.094435 \\
1 & 0.903476 & 1.133428 & 1.445393 \\
1 & -2.160808 & 1.257656 & -0.289901 \\
1 & 0.202643 & -1.962199 & -0.236778 \\
1 & -0.275081 & 2.586788 & -0.762061 \\
\hline
\end{tabular}

\begin{tabular}{cccc}
\hline \multicolumn{4}{c}{ 5-fluorouracil } \\
\hline $\begin{array}{c}\text { Atom } \\
\text { Number }\end{array}$ & $\mathrm{x}$ & $\mathrm{y}$ & $\mathrm{z}$ \\
\hline 7 & -1.193108 & 0.888277 & -0.471002 \\
6 & -1.313188 & -0.435051 & 0.055839 \\
7 & -0.156112 & -1.141720 & 0.047623 \\
6 & 1.138734 & -0.609572 & -0.212053 \\
6 & 1.197688 & 0.889685 & -0.142315 \\
6 & -0.037714 & 1.452213 & -0.726419 \\
8 & -2.395063 & -0.835398 & 0.360119 \\
8 & 2.043222 & -1.372978 & -0.376286 \\
9 & 0.987331 & 1.125525 & 1.243572 \\
1 & -2.071343 & 1.317989 & -0.696863 \\
1 & -0.236540 & -2.114913 & 0.257683 \\
1 & -0.078589 & 2.373459 & -1.282253 \\
\hline
\end{tabular}

Table S13. Cartesian coordinates of the CASSCF $(8 / 8) / 6-31 G(d)$ optimized structure of thymine.

\begin{tabular}{cccc}
\hline \multicolumn{4}{c}{ thymine } \\
\hline $\begin{array}{c}\text { Atom } \\
\text { Number }\end{array}$ & $\mathrm{x}$ & $\mathrm{y}$ & $\mathrm{z}$ \\
\hline 6 & -0.063553 & 1.318783 & -0.903864 \\
7 & -1.226106 & 0.917070 & -0.462744 \\
6 & -1.338667 & -0.406286 & 0.098284 \\
7 & -0.218368 & -1.161928 & -0.043504 \\
6 & 1.116348 & -0.654641 & -0.269903 \\
6 & 1.148986 & 0.827755 & -0.276619 \\
8 & -2.402496 & -0.765524 & 0.500836 \\
8 & 2.001942 & -1.457581 & -0.274615 \\
6 & 1.144423 & 1.250916 & 1.219357
\end{tabular}




\begin{tabular}{ccc}
-2.104835 & 1.324198 & -0.730181 \\
-0.320626 & -2.126334 & 0.194679 \\
-0.105348 & 2.025451 & -1.724861 \\
1.013164 & 2.324679 & 1.312931 \\
0.413427 & 0.774100 & 1.881089 \\
2.127365 & 1.025193 & 1.618126 \\
\hline
\end{tabular}

\section{Full references}

(60) Gaussian Development Version, Revision D.02, Frisch, M. J.; Trucks, G. W.; Schlegel, H. B.; Scuseria, G. E.; Robb, M. A.; Cheeseman, J. R.; Montgomery, Jr., J. A.; Vreven, T.; Kudin, K. N.; Burant, J. C.; Millam, J. M.; Iyengar, S. S.; Tomasi, J.; Barone, V.; Mennucci, B.; Cossi, M.; Scalmani, G.; Rega, N.; Petersson, G. A.; Nakatsuji, H.; Hada, M.; Ehara, M.; Toyota, K.; Fukuda, R.; Hasegawa, J.; Ishida, M.; Nakajima, T.; Honda, Y.; Kitao, O.; Nakai, H.; Klene, M.; Li, X.; Knox, J. E.; Hratchian, H. P.; Cross, J. B.; Bakken, V.; Adamo, C.; Jaramillo, J.; Gomperts, R.; Stratmann, R. E.; Yazyev, O.; Austin, A. J.; Cammi, R.; Pomelli, C.; Ochterski, J. W.; Ayala, P. Y.; Morokuma, K.; Voth, G. A.; Salvador, P.; Dannenberg, J. J.; Zakrzewski, V. G.; Dapprich, S.; Daniels, A. D.; Strain, M. C.; Farkas, O.; Malick, D. K.; Rabuck, A. D.; Raghavachari, K.; Foresman, J. B.; Ortiz, J. V.; Cui, Q.; Baboul, A. G.; Clifford, S.; Cioslowski, J.; Stefanov, B. B.; Liu, G.; Liashenko, A.; Piskorz, P.; Komaromi, I.; Martin, R. L.; Fox, D. J.; Keith, T.; Al-Laham, M. A.; Peng, C. Y.; Nanayakkara, A.; Challacombe, M.; Gill, P. M. W.; Johnson, B.; Chen, W.; Wong, M. W.; Gonzalez, C.; and Pople, J. A.; Gaussian, Inc., Wallingford CT, 2005.

\section{SI references}

(1) Adamo, C.; Scuseria, G. E.; Barone, V. J. Chem. Phys. 1999, 111, 2889-2899.

(2) Staroverov, V. N.; Scuseria, G. E.; Tao, J.; Perdew, J. P. J. Chem. Phys. 2003, 119, 12129-12137.

(3) Boese, A. D.; Martin, J. M. L.; Handy, N. C. J. Chem. Phys. 2003, 119, 3005-3014.

(4) Zhao, Y.; Truhlar, D. G. J. Chem. Theory and Comput. 2005, 1, 415-432.

(5) Siano, D. B.; Metzler, D. E. J. Chem. Phys. 1969, 51, 1856-1861.

(6) Maroncelli, M.; Fleming, G. R. J. Chem. Phys. 1987, 86, 6221.

(7) Gustavsson, T.; Cassara, L.; Gulbinas, V.; Gurzadyan, G.; Mialocq, J.-C.; Pommeret, S.; Sorgius, M.; van der Meulen, P. J. Phys. Chem. A 1998, 102, 4229-4245. 